rest of clinical and immunological features were similar to previously described in other series.

References:

[1] Amezcua Guerra LM. Overlap between systemic lupus erythematosus and rheumatoid arthritis: is it real or just an illusion? J Rheumatol 2009; 36: 4-6.

[2] Li J, Wu H, Huang X, Xu D, Zheng W, Zhao Y, et al. Clinical analysis of 56 patients with rhupus syndrome: manifestations and comparisons with systemic lupus erythematosus. Medicine 2014; 93(10).

[3] Simón JA, Granados J, Cabiedes J, Ruiz Morales J, Alcocer Varela J. Clinical and immunogenetic characterization of Mexican patients with rhupus. Lupus 2002; 11: 287-292

[4] Tani C, D’Aniello D, Delle Sedie A, Carli L, Cagnoni M, Possemato N, et al. Rhupus syndrome: assessment of its prevalence and its clinical and instrumental characteristics in a prospective cohort of 103 SLE patients. Autoimmun Rev 2013; 12: 537-541.

Disclosure of Interests: None declared

DOI: 10.1136/annrheumdis-2020-eular.4972

\section{AB1045 $\quad$ CLINICAL, ANALYTICAL AND RADIOLOGICAL CHARACTERISTICS OF A COHORT OF PATIENTS WITH SARCOIDOSIS.}

I. Madroñal García ${ }^{1}$, C. Aguilera $\operatorname{Cros}^{1}$, L. Mendez Diaz ${ }^{1}{ }^{1}$ Hospital Universitario Virgen del Rocío, Reumatologia, Sevilla, Spain

Background: Sarcoidosis is a systemic disease whose etiology is unknown. It is characterized by the formation of granulomas in any tissue of the organism. Ganglionic, pulmonary and cutaneus involvement is the most prevalent. Objectives:

1. Describe clinical characteristics of a cohort of patients with sarcoidosis diagnosed.

2. Define the association between the ACE's number at diagnosis, radiological lung stage, treatment and course of disease.

3. Evaluate if the extrapulmonary involvement is related to the course of the disease.

Methods: Descriptive retrospective study of patients with $\mathrm{S}$ diagnosis treated in our Hospital in 2019. Data were obtained by reviewing medical records. Chisquare tests and parametric tests have been used to establish the differences described in the objectives.

Results: 102 patients diagnosed with sarcoidosis have been included, (51\% females) with an average age of $56 \pm 11$ years. Suspected diagnosis at the onse of disease was S in $70.6 \%$ of patients, followed by suspected lymphoma $(20.6 \%)$ The average time for the definitive diagnosis of $S$ was 9.5 months. $70.6 \%$ of the patients had elevated ACE titles at the beginning. Regarding the clinical manifestations, $18.6 \%$ of the patients presented fever at the beginning and $66.7 \%$ extrathoracic clinical manifestations. $72.5 \%$ have lymph node adenopathies, and in $91 \%$ there is thoracic involvement (the most frequent pulmonary stage is stage II). A biopsy was performed in $84.3 \%$ of the patients, the lung biopsy being the most performed (52.3\%). $88.2 \%$ of patients received corticosteroid treatment at the onset of the disease (currently under treatment with corticosteroids $37.3 \%$ ). $50 \%$ of patients are treated with immunosuppressants, Methotrexate was the most used. 5 patients are treated with biological therapy (AntiTNF).

Regarding the course of the disease, $51 \%$ of the patients have a chronic course $45.1 \%$ are in remission and $3.9 \%$ have suffered a relapse of the disease. In this study, no significant relationship was found between the ACE values at the onset of the disease, the pulmonary stage and the course of the disease.

According to our data, patients presenting with extrathoracic clinical manifestations need more frequently corticosteroid treatment $(p=0.017)$ and immunosuppressive treatment $(p=0.001)$ with respect to patients who do not have an extrathoracic clinic. In addition, patients with an extrathoracic clinic present more frequently a chronic course of the disease than those who do not $(p=0.019)$

Conclusion: The results described in this study are similar to those found in the literature. The differences found can be explained because patients presenting with extrathoracic clinical manifestations have a more complicated management and need more treatment than those with only pulmonary involvement, even patients with radiological stage I do not usually need treatment, only surveillance.

Disclosure of Interests: None declared

DOI: 10.1136/annrheumdis-2020-eular.6414

\section{AB1046 JOINT MANIFESTATIONS IN A COHORT OF PATIENTS WITH SARCOIDOSIS IN A THIRD LEVEL HOSPITAL.}

I. Madroñal García ${ }^{1}$, C. Aguilera $\operatorname{Cros}^{1}$, L. Mendez Diaz ${ }^{1}{ }^{1}$ Hospital Universitario Virgen del Rocio, Reumatologia, Sevilla, Spain
Background: Sarcoidosis (S) is a systemic granulomatous disease of unknown etiology, which most frequently affects the ganglion, lung and skin, although it can affect other organs, including the musculoskeletal system.

Objectives:

Describe the clinical, analytical and radiological characteristics of patients diagnosed with $\mathrm{S}$ presenting joint manifestations.

To assess the association between patients who have joint manifestations and the use of corticosteroids (C) and immunosuppressants (IS), with respect to those without joint involvement.

Methods: Retrospective descriptive study of patients with diagnosis of $\mathrm{S}$ with joint manifestations, treated in our Hospital from 2017 to 2019. Data were obtained by reviewing medical records. Chi square tests and Fisher's exact test have been performed to establish the differences described in the objectives.

Results: From a database of 102 patients with S, 18 presented joint manifestations ( $50 \%$ women), with a mean age of $57 \pm 6$ years. Of these patients, 4 $(22.2 \%)$ have presented positive ANA. Regarding the clinic, 3 patients presented the association of polyarthritis and bilateral ankle swelling, 8 patients presented with polyarthritis, 3 monoatritis and 4 patients presented bilateral ankle swelling $61.1 \%$ had fever at the onset of the disease.

14 patients $(77.8 \%)$ had high ACE values at the onset of the disease, without presenting significant differences with respect to all patients diagnosed with $S$ who do not have joint involvement.

All patients received treatment with $\mathrm{C}$ and 10 patients $(55.5 \%)$ needed an IS treatment, finding no differences with respect to patients who do not have joint involvement $(p=0.92)$

On the course of the disease, the majority of patients with joint involvement have a chronic course $(72.2 \%)$. Nor were significant differences found when compared with patients who have no joint involvement $(p=0.73)$.

Conclusion: Patients with joint involvement in our study have been $17.6 \%(18)$ an approximate result to that described in the literature (over $10 \%$ ), although our result may be increased by the fact that the patients who are followed in Rheumatology present or have presented joint involvement. No significant differences were found between patients with $\mathrm{S}$ who presented joint involvement and those who did not, with respect to the initial ACE values, treatment and disease course. Prospective, multicenter and larger sample studies are necessary to better understand these associations.

Disclosure of Interests: None declared

DOI: 10.1136/annrheumdis-2020-eular.6444

\section{AB1047 \\ IGG4-RELATED DISEASE PRESENTATION REQUIRES ADMISSION TO THE EMERGENCY DEPARTMENT IN THE MAJORITY OF CASES}

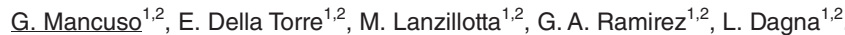
${ }^{1}$ Università Vita-Salute San Raffaele, Milano, Italy; ${ }^{2}$ San Rafael Hospital, Unit of Immunology, Rheumatology, Allergy and Rare Diseases, Milano, Italy

Background: IgG4-related disease (IgG4-RD) is generally considered a chronic fibro-inflammatory condition with insidious presentation and subclinical course. Our clinical experience, however, suggests that a sizable proportion of patients experience multiple accesses to the emergency department (ED), either at dis ease onset or during the disease course.

Objectives: In the present study we aimed (i) to assess the prevalence of acute manifestations of IgG4-RD at disease onset requiring referral to the $E D$, and (ii) to calculate the diagnostic delay from the initial acute presentation.

Methods: We revised our database and identified patients admitted to the ED because of symptoms lately attributed to IgG4-RD onset (Group 1) and those that were referred to our outpatient clinic without previous urgent manifestations (Group 2). Acute manifestations were clustered based on the anatomical distric affected by IgG4-RD. Epidemiological, clinical, and serological features of Group 1 and Group 2 were compared.

Results: The study included 141 patients with IgG4-RD. 76 (54\%) presented to the ED at disease onset. The most common clinical manifestations requiring admission to the ED were jaundice (53\%), abdominal pain $(41 \%)$, and feve (10\%). Gastrointestinal involvement was the most frequent cause of referral to the ED ( $71 \%$ of cases), followed by involvement of the retroperitoneum $(14.5 \%)$, and of the nervous system (6.6\%). Pancreato-biliary involvement was significantly more frequent in Group 1. Head, neck, salivary and lacrimal gland involvement was more frequent in Group 2. The diagnostic delay was significantly shorter in Group 1 than in

Group 2.

Conclusion: Clinical manifestations associated with IgG4-RD onset require referral to the ED in the majority of cases. This finding contrasts with the genera view of IgG4-RD as a condition with non-acute presentation. 
References:

[1] Bledsoe JR, Della-Torre E, Rovati L, Deshpande V. IgG4-related disease: review of the histopathologic features, differential diagnosis, and therapeutic approach. APMIS. 2018;126:459-476.

[2] Della-Torre E, Lanzillotta M, Doglioni C. Immunology of IgG4-related disease. Clin Exp Immunol.2015;181:191-206.

[3] Lanzillotta M, Campochiaro C, Trimarchi M, Arrigoni G, Gerevini S, Milani R, et al. Deconstructing IgG4-related disease involvement of midline structures: Comparison to common mimickers. Mod Rheumatol. 2017;27:638-645.

[5] Della-Torre E, Stone JH. "How I manage" IgG4-Related Disease. J Clin Immunol. 2016;36:754-763.

[6] Perugino CA, Mattoo H, Mahajan VS, Maehara T, Wallace ZS, Pillai S, et al. Emerging Treatment Models in Rheumatology: IgG4-Related Disease: Insights Into Human Immunology and Targeted Therapies. Arthritis Rheumatol. 2017;69:1722-1732.

[7] Stone JH, Zen Y, Deshpande V. IgG4-related disease. N Engl J Med. 2012;366:539-51.

[8] Kamisawa T, Zen Y, Pillai S, Stone JH. IgG4-related disease. Lancet. 2015;385:1460-71

[9] Umehara H, Okazaki K, Masaki Y, Kawano M, Yamamoto M, Saeki T, et al. Comprehensive diagnostic criteria for IgG4-related disease (IgG4-RD), 2011. Mod Rheumatol. 2012;22:21-30.

Disclosure of Interests: Gaia Mancuso: None declared, Emanuel Della Torre: None declared, Marco Lanzillotta: None declared, Giuseppe Alvise Ramirez: None declared, Lorenzo Dagna Grant/research support from: The Unit of Immunology, Rheumatology, Allergy and Rare Diseases (UnIRAR) received unresctricted research/educational grants from Abbvie, Bristol-Myers Squibb, Celgene, Janssen, Merk Sharp \& Dohme, Mundipharma Pharmaceuticals, Novartis, Pfizer, Roche, Sanofi-Genzyme, and SOBI., Consultant of: Prof Lorenzo Dagna received consultation honoraria from Abbvie, Amgen, Biogen, Bristol-Myers Squibb, Celltrion, Novartis, Pfizer, Roche, Sanofi-Genzyme, and SOBI.

DOI: 10.1136/annrheumdis-2020-eular.2919

\section{AB1048 $\quad$ RHUPUS SYNDROME IN A TERTIARY HOSPITAL}

1. Martínez Cordellat ${ }^{1}$, R. Gonzalez Mazario', M. De la Rubia Navarro', C. Pávez Perales ${ }^{1}$, S. Leal Rodriguez ${ }^{1}$, J. Ivorra Cortés ${ }^{1}$, I. Chalmeta Verdejo ${ }^{1}$, E. Grau García ${ }^{1}$, C. Alcañiz Escandell ${ }^{1}$, J. J. Fragio-Gil ${ }^{1}$, L. Gonzalez Puig ${ }^{1}$, R. Negueroles Albuixech ${ }^{1}$, J. E. Oller Rodríguez ${ }^{1}$, F. M. Ortiz Sanjuan ${ }^{1}$, E. Vicens Bernabeu ${ }^{1}$, C. Nájera Herranz ${ }^{1}$, I. Cánovas Olmos ${ }^{1}$, J. A. Román Ivorra ${ }^{1} .{ }^{1}$ Rheumatology Department. HUP La Fe, Valencia, Spain

Background: Rhupus syndrome (RhS) is a rare combination of rheumatoid arthritis (RA) and systemic lupus erythematosus (SLE). Different studies describe RhS cases that begin with erosive arthritis and the presence of rheumatoid factor (RF) and/or anti CCP and then the SLE symptoms.

Objectives: Despite the fact that RhS shows a low prevalence, it would be useful to know clinical characteristics of RhS patients since their therapy and outcome differ from those having RA or SLE alone.

Methods: Retrospective study with systematic revision of electronic clinical records of RhS patients was performed. Demographic, clinical and immunological data were collected.

Results: Eight RhS patients were included (all fulfilled SLICC 2012 criteria for SLE and ACR 2010 for RA). Mean age was 67.3 (45-84) years (7 were female). In 3 cases RA was the first diagnosis with a mean evolution of 4.5 years until SLE diagnosis. In contrast, in 5 cases SLE was the first diagnosis with a mean evolution of 7.2 years until RA diagnosis. Photosensitivity and arthritis were the predominant clinical manifestations. One patient presents pericarditis and other case showed rheumatoid nodules in elbows. Renal, pulmonary or neurological affection was no reported.

4 patients were under biological/JAK inhibitors therapies (2 abatacept, 1 rituximab and 1 baricitinib) with favorable response of treatment.

Conclusion: In contrast to other series, only the $37.5 \%$ of our RhS cases begins with polyarticular seropositive arthritis. The $62.5 \%$ started with SLE symptoms as haematological alterations, cutaneous and serological manifestation, and showed longer progression to have polyarticular affection. Thus, RhS diagnosis is earlier in patients that begin with RA symptoms. 4 RhS patients were refractory to DMARd treatments, where biological/JAK inhibitors therapies are needed. Disclosure of Interests: None declared

DOI: 10.1136/annrheumdis-2020-eular.3468

\section{AB1049 CLINICAL SPECTRUM AND THERAPEUTIC MANAGEMENT OF AUTO-IMMUNE MYELOFIBROSIS: A NATION-WIDE STUDY OF 30 CASES}

$\underline{\text { P. Mertz }}^{1}$, E. Chalayer ${ }^{2}$, J. Sibilia ${ }^{1}$, J. E. Gottenberg ${ }^{1}$, A. S. Korganow ${ }^{3}$ L. Arnaud', T. Martin ${ }^{3}$. 'Service de Rhumatologie, Hôpitaux Universitaires de Strasbourg, Laboratoire d'ImmunoRhumatologie Moléculaire, INSERM UMR_S1109, Centre National de Référence des Maladies Systémiques et Autoimmunes Rares Est Sud-Ouest (RESO), Université de Strasbourg, Strasbourg, France; ${ }^{2}$ Institut de Cancérologie Lucien Neuwirth, Saint Priest en Jarez, France; ${ }^{3}$ Service de Médecine Interne et d'Immunologie Clinique Hôpitaux Universitaires de Strasbourg, Centre National de Référence des Maladies Systémiques et Autoimmunes Rares Est Sud-Ouest (RESO), Université de Strasbourg, Strasbourg, France

Background: Little is known about autoimmune myelofibrosis (AIMF), a rare entity that can occur alone or in association with another autoimmune disease (AID) and is responsible for bone marrow (BM) failure and life-threatening complications.

Objectives: We conducted a nationwide retrospective observational study of AIMF cases to better characterize the epidemiology, clinical presentation and evolution of this rare entity.

Methods: The aim of the study was to analyze the characteristics of AIMF and the nature and indication of treatments currently used. Response to treatment was evaluated by the revised Tefferi et al. response criteria.

Results: Among 30 cases of AIMF, the sex ratio (F/M) was 4:1 and the median age at diagnosis was 37 years (interquartile range 30-49). AIMF was diagnosed after the onset of an associated AID in 12 cases and concomitant to an AID in the remaining 18 cases. The most frequently associated AID was systemic lupus erythematous, followed by Sjögren syndrome. All cases consisted of reticulin fibers, and no collagen fibrosis was described. More than $50 \%$ of cases showed complete response after first-line therapy, with glucocorticoids (GC) in 28 cases Half of the cases had treatment complications mainly related to GC therapy. Conclusion: Diagnosis of AIMF remains challenging in the absence of a validated set of diagnosis criteria, and must always be searched in the presence of hematological abnormalities at onset or during follow-up of AID. Clinical context, search for mutations and pathology findings can help differentiating this rare disease from a clonal pathology. GC is currently an effective first-choice therapy for AIMF, but a high rate of GC dependency and long-term complications indicate the need to find new sparing drugs.

Disclosure of Interests: PHILIPPE MERTZ: None declared, Emilie Chalayer: None declared, Jean Sibilia: None declared, Jacques-Eric Gottenberg Grant/research support from: BMS, Pfizer, Consultant of: BMS, Sanofi-Genzyme, UCB, Speakers bureau: Abbvie, Eli Lilly and Co., Roche, Sanofi-Genzyme, UCB, Anne-Sophie Korganow: None declared, Laurent Arnaud: None declared, Thierry Martin: None declared DOI: 10.1136/annrheumdis-2020-eular.998

$\mathrm{AB} 1050$

CLINICAL IMPLICATIONS OF ULTRASONOGRAPHY (US) IN DIAGNOSIS AND MONITORING DISEASE ACTIVITY OF RELAPSING POLYCHONDRITIS (RP) AND COMPARATIVE INVESTIGATION BY US BETWEEN AURICLE OF RP, REPEATED TRAUMA, CELLULITIS AND HEALTHY SUBJECT

H. Nishikawa ${ }^{1}$, Y. Taniguchi ${ }^{1}$, M. Ogasawara ${ }^{1}$, S. Inotani ${ }^{1}$, E. Amano ${ }^{1}$,

T. Matsumoto ${ }^{1}$, K. Hamada-Ode ${ }^{1}$, Y. Shimamura ${ }^{1}$, T. Horino ${ }^{1}$, S. Fujimoto ${ }^{1}$, Y. Terada ${ }^{1} .{ }^{1}$ Kochi Medical School Hospital, Nankoku, Japan

Objectives: To assess the clinical implications of ultrasonography (US) in monitoring disease activity and diagnosis of relapsing polychondritis (RP).

Methods: Firstly, auricular $(n=5)$ and nasal $(n=1)$ chondritis of six patients with RP were assessed by US before and after treatments. The relationship between US findings and serum markers were evaluated. Moreover, the comparisons of US findings between the auricle of patients with RP $(n=5)$, repeated trauma $(n=5)$, cellulitis $(n=2)$ and healthy subjects $(n=5)$ were also assessed.

Results: US finding before treatment showed low-echoic swollen auricular and nasal cartilage with increased power Doppler signals (PDS) in all cases of RP. US findings corresponded to biopsy findings. After treatment, the swollen ear and nose completely resolved. Then, US findings also showed dramatic reductions in swollen cartilage with the decrease in PDS. Although serum markers completely improved, US finding remained in 1 of 6 cases, and this case showed flare due to PSL tapering. Finally, RP could be differentiated from the damage of repeated trauma and cellulitis by the presence or absence of PDS and subperichondrial serous effusion

Conclusion: US of auricular and nasal cartilage in RP possibly facilitates evaluation of auricular lesions and monitoring of disease activity, especially when we consider the treatment response and the timing of drug tapering.

Disclosure of Interests: None declared

DOI: 10.1136/annrheumdis-2020-eular.4158

\section{AB1051 KIKUCHI FUJIMOTO DISEASE, IS IT SLE?}

J. Camins-Fàbregas ${ }^{1}$, V. Ortiz-Santamaria ${ }^{1}$, N. Busquets-Pérez ${ }^{1}$, A. Cuervo ${ }^{1}$, I. Cañas Alcántara ${ }^{2}$, R. Acal ${ }^{2}$, E. Hadad-Casorelli ${ }^{2}$, A. Guilabert ${ }^{3}$, J. Sola $^{3}$. ${ }^{1}$ Hospital General de Granollers, Rheumatology, Granollers, Spain; ${ }^{2}$ Hospital General de Granollers, Internal Medicine, Granollers, Spain; ${ }^{3}$ Hospital General de Granollers, Dermatology, Granollers, Spain 